\title{
Transcatheter mitral valve repair using the Cardiobandß system: Histopathological insights in device ingrowth and biocompatibility
}

\author{
Martin Schmiady ${ }^{1}$, Mathias van Hemelrijck ${ }^{1}$, Maurizio Taramasso ${ }^{2}$, Juri Sromicki ${ }^{1}$, Carlos \\ Mestres $^{3}$, and Matthias Sigler ${ }^{4}$ \\ ${ }^{1}$ UniversitatsSpital Zurich \\ ${ }^{2}$ Hirslanden Klinik Hirslanden \\ ${ }^{3}$ University Hospital Zurich \\ ${ }^{4}$ Georg -August University,
}

June 23, 2021

\begin{abstract}
Surgical implantation of a complete or incomplete ring to reduce the valve annulus and improve leaflet coaptation is the mainstay of mitral valve surgery. The Cardioband@ system (Edwards Lifesciences, Irvine, CA, USA) was designed to address the pathophysiological mechanism of annular dilatation through a catheter-based approach. We present the histopathological workup

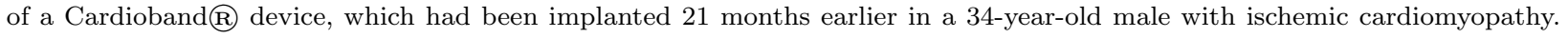
Device examination demonstrate a well-positioned and securely anchored device. The described tissue reactions may have an impact on choice of device and timing in case of re-do surgery.
\end{abstract}

\section{Transcatheter mitral valve repair using the Cardioband ${ }^{\circledR}$ system: Histopathological insights in device ingrowth and biocompatibility}

Martin O. Schmiady ${ }^{1 *}$; Mathias Van Hemelrijck ${ }^{1}$, Maurizio Taramasso ${ }^{2}$; Juri Sromicki ${ }^{1}$, Carlos A. Mestres ${ }^{1}$, and Matthias Sigler ${ }^{3}$

${ }^{1}$ Division of Cardiac Surgery, University Heart Center, University Hospital Zurich, Raemistrasse 100, 8091 Zurich, Switzerland

${ }^{2}$ Heart Center Hirslanden Zurich, Witellikerstrasse 36, 8008 Zurich, Switzerland

${ }^{3}$ Pediatric Cardiology and Intensive Care Medicine, Georg-August University, Robert-Koch-Strasse 40, 37075 Goettingen, Germany

${ }^{*}$ Corresponding author: Martin O. Schmiady. Division of Cardiac Surgery, University Heart Center, University Hospital Zurich, Raemistrasse 100, CH-8091 Zurich, Switzerland.

Tel: +41 4425595 82; fax: +41 4425544 67, e-mail: martinoliver.schmiady@usz.ch

Keywords: Cardioband ${ }^{\circledR}$, transcatheter mitral valve repair, mitral annuloplasty, mitral regurgitation, biocompatibility, Tissue reaction

Surgical implantation of a complete or incomplete ring to reduce the valve annulus and improve leaflet coaptation is the mainstay of mitral valve surgery. The Cardioband ${ }^{\circledR}$ system (Edwards Lifesciences, Irvine, CA, USA) was designed to address the pathophysiological mechanism of annular dilatation through a catheter-based approach ${ }^{1}$. We present the histopathological workup of a Cardioband ${ }^{\circledR}$ device, which had 
been implanted 21 months earlier in a 34-year-old male with ischemic cardiomyopathy and severe functional mitral regurgitation. Starting at the anterolateral commissure, 17 anchors were implanted under general anaesthesia, echo- and fluoro guidance (Panels A and B). A significant reduction in mitral valve regurgitation from severe to trivial was achieved (Panels C and D). Even though the patient's clinical symptoms improved, he later was listed for transplantation due to the severely impaired left ventricular function (LVEF 15\%).

After surgical removal, the device was preserved in formalin and later embedded in a synthetic resin (methylmethacrylate, Technovit 9100, KULZER\&Co, Wehrheim, Germany), hardened, and subsequently sectioned in slices of $0.8 \mathrm{~mm}$ using a diamond cutter. These slices were ground down to 10-30 $\mu \mathrm{m}$ using a rotational grinder. Standard staining was performed with Richardson blue. An extended histopathological work-up was performed ${ }^{2}$. On gross examination, the Cardioband ${ }^{\circledR}$ was intact. All anchors were well positioned and firmly attached to the annulus (Panel E). This was also confirmed by histology (Panel F). No superficial deposit of thrombus material was detected. A thin layer of endothelialized pseudointima had formed on the surface of the device.

In summary, we can demonstrate a well-positioned and securely anchored device. The architecture of the valve tissue and the subvalvular apparatus were preserved thus allowing for the combination with other percutaneous reconstructive methods. Screws were firmly anchored and endothelialized, making explantation in case of redo surgery challenging.

\section{References}

1. Miller M, Thourani VH, Whisenant B. The Cardioband transcatheter annular reduction system. Ann Cardiothorac Surg . 2018;7(6):741-747. doi:10.21037/acs.2018.10.10.

2. Sigler M, Paul T, Grabitz RG. Biocompatibility screening in cardiovascular implants. Z Kardiol . 2005;94(6):383-391. doi:10.1007/s00392-005-0231-4.

\section{Figure 1}

\section{Hosted file}

image1.emf available at https://authorea.com/users/314227/articles/527453-transcathetermitral-valve-repair-using-the-cardioband-system-histopathological-insights-in-deviceingrowth-and-biocompatibility

Fig.1 A and B: Placement of the Cardioband ${ }^{\circledR}$ using fluoro- and TEE guidance. C and D: Final result demonstrating a significant reduction in mitral valve regurgitation. E: Intraoperative view on the well positioned Cardioband ${ }^{\circledR} 21$ months after implantation. F: Histology demonstrating a well-fitted and securely anchored device.

\section{Compliance with ethical standards:}

All procedures performed in this case were in accordance with the ethical standards of the institutional and/or national research committee and with the 1964 Helsinki declaration and its later amendments or comparable ethical standards. Informed consent was obtained from the patient included in this work. The IRB approved this case Study.

\section{Declaration of Conflicting Interests}

All authors declared no potential conflict of interest with respect to the research, authorship, and/or publication of this article.

\section{Funding}

None of the authors received financial support for research, authorship, and/or publication of this article. 